\title{
Paclitaxel synergizes with exposure time adjusted CD22- targeting immunotoxins against B-cell malignancies
}

\author{
Fabian Müller ${ }^{1,2}$, Stephanie Stookey ${ }^{1,3}$, Tyler Cunningham ${ }^{1,4}$ and Ira Pastan ${ }^{1}$ \\ ${ }^{1}$ Laboratory of Molecular Biology, Center for Cancer Research, National Cancer Institute, National Institutes of Health, \\ Bethesda, MD, USA \\ 2 Department of Hematology and Oncology, University Hospital Erlangen, Erlangen, Germany \\ ${ }^{3}$ MD Program, University of North Caroline, Chapel Hill, NC, USA \\ ${ }^{4}$ MD/PhD Program, University of Miami, Miller School of Medicine, Miami, FL, USA \\ Correspondence to: Ira Pastan, email: pastani@mail.nih.gov \\ Keywords: CD22-targeted immunotoxin, mantle cell lymphoma, targeted therapy, combination therapy, paclitaxel \\ Received: January 18, 2017 \\ Accepted: February 18, 2017 \\ Published: March 11, 2017
}

Copyright: Müller et al. This is an open-access article distributed under the terms of the Creative Commons Attribution License (CC-BY), which permits unrestricted use, distribution, and reproduction in any medium, provided the original author and source are credited.

\section{ABSTRACT}

CD22-targeted recombinant immunotoxins (rIT) are active in hairy cell leukemia or acute lymphoblastic leukemia (ALL), but not in mantle cell lymphoma (MCL) patients. The goal was to enhance rIT efficacy in vivo and to define a strong combination treatment. Activity of Moxetumomab pasudotox (Moxe) and LR combined with paclitaxel was tested against MCL cell lines in vitro and as bolus doses or continuous infusion in xenograft models. In the KOPN-8 ALL xenograft, Moxe or paclitaxel alone was active, but all mice died from leukemia; when combined, $60 \%$ of the mice achieved a sustained complete remission. Against MCL cells in vitro, LR was more active than Moxe and the cells had to be exposed to rIT for more than 24 hours for them to die. To maintain high blood levels in vivo, LR was administered continuously by 7-day pumps achieving a well-tolerated steady plasma concentration of $45 \mathrm{ng} / \mathrm{ml}$. In JeKo-1 xenografts, continuously administered LR was 14-fold more active than bolus doses and the combination with paclitaxel additionally improved responses by 135 -fold. Maintaining high rIT-plasma levels greatly improves responses in the JeKo-1 model and paclitaxel substantially enhances bolus and continuously infused rIT, supporting a clinical evaluation against B-cell malignancies.

\section{INTRODUCTION}

We have developed recombinant immunotoxins (rIT) which consist of an antibody fragment fused to a truncated Pseudomonas exotoxin A [1]. The first CD22targeting rIT BL22 (CAT-3888) is active against B-cell non-Hodgkin lymphoma (B-NHL) cell lines in vitro [2] and achieves an objective response rate of $81 \%$ in patients with hairy cell leukemia (HCL) [3]. However, BL22 fails to produce objective responses in pediatric B-cell acute lymphoblastic leukemia (B-ALL) [4] or mantle cell lymphoma (MCL) [3]. The affinity matured BL22variant HA22 or Moxetumomab pasudotox (Moxe) [5] is 10 -fold more potent than BL22 in vitro and achieves an overall response rate of $86 \%$ in HCL patients [6] and of $33 \%$ in pediatric B-ALL [7]. HCL patients treated with
Moxe regularly develop neutralizing antibodies which likely reduce the anti-leukemic activity in these patients [6]. To decrease immunogenicity, we generated HA22PE24 (named LR) [8] which lacks domain II and therefore major B- [9] and T-cell epitopes [10]. In addition, LR is more active than Moxe or BL22 against various ALL and lymphoma cell lines and against patient-derived chronic lymphocytic leukemia (CLL) [8]. LR has not yet been developed clinically.

MCL is a rare subtype of B-NHL of $6 \%$ [11]. New treatment options include the Burton's Tyrosine kinase (BTK)-inhibitor Ibrutinib [12, 13], the proteasome inhibitor Bortezomib [14-16], the immune-modulator Lenalidomide [17-19], and the mTORC-inhibitor Temsirolimus $[13,20]$ which are active in patients with relapsed/refractory MCL; various combinations of these 
small molecules with current standard treatment regimens are in clinical testing [11, 21-23]. Despite recent progress in treatment options and the introduction of intensified chemotherapy regimens with autologous stem cell transplantation leading to a doubling of progressionfree survival of MCL patients over the past two decades, most patients still relapse and MCL remains an incurable disease [22].

We recently showed that the activity of CD22targeting rIT against ALL cells in vitro and in vivo improved substantially when the cells were exposed longer to CD22-targeting rIT [24]. Because rITs have a short plasma half-life in mice [8] and men [7], blood levels fall quickly after a bolus dose. The rIT exposure time of ALL cells growing in murine bone marrow (BM) achieved by bolus doses was too short for them to die; when high rIT blood levels were maintained by repeated injections, in vivo efficacy was greatly improved [24].

Mesothelin-targeting rITs are enhanced by various small molecules [25-27] or the chemotherapeutic drug paclitaxel [28-30]. Paclitaxel enhances the efficacy of bolus doses of mesothelin-targeted rIT in vivo [28, 29, 31 , which was translated in a new clinical trial testing the combination (NCT02810418). Whether in vivo efficacy of CD22-targeting rIT is enhanced by paclitaxel has not been determined previously.

Here, we tested whether the addition of paclitaxel would improve treatment outcome in the KOPN-8 xenograft model [24]. To extend our studies toward MCL, various cell lines were tested in vitro and LR was found to be more active than Moxe. The time that MCL cells needed to be exposed to either rIT for them to die varied largely from hours to days. To enable continuous drug delivery in vivo by 7-day osmotic pump, the rIT-formulation buffer was optimized to ensure protein stability, which then allowed for the comparison of bolus dose and continuous administration and finally the combination with paclitaxel in a newly established systemic JeKo-1 xenograft model.

\section{RESULTS}

\section{Paclitaxel/rIT-combination achieves durable remissions in an ALL xenograft model}

To search for combination therapies which enhance the in vivo efficacy of CD22-targeting rITs, we used our recently developed KOPN-8 xenograft model [24]. After i.v. injection, the KOPN-8 cells grow in the murine BM and three bolus doses of Moxe every other day (QOD) reduce the KOPN-8 BM infiltration below the detection limit of flow cytometry. However, all mice relapse and die from leukemia [24]. To track treatment responses in real time, KOPN-8 cells were transduced with luciferase and green fluorescent protein (GFP) and single cloned.
After i.v. injection, the disease burden was measured by determining the bioluminescence. In this KOPN-8 model, the combination of paclitaxel and Moxe improved responses greatly (Figure 1A). Before start of treatment on day 7, all mice showed a similar bioluminescence signal. In accordance with the known KOPN-8 BM infiltration [24], the bioluminescence was found in close proximity to bones. Untreated mice progressed rapidly as shown by a steep increase in bioluminescence. For mice receiving a single dose of $25 \mathrm{mg} / \mathrm{kg}$ paclitaxel i.p. on day 7 bioluminescence increased between days 7 and 19 , but was lower than the bioluminescence of control mice on day 19. Three i.v. bolus doses of $0.4 \mathrm{mg} / \mathrm{kg}$ Moxe QOD given from day 8 resulted in an abrogation of the bioluminescence signal on day 19 but the bioluminescence recurred in all these mice indicating expected leukemia relapse. The mice which had received the combination of Moxe and paclitaxel showed an abrogation of the bioluminescence on day 19 . In mice 2,6 , and 9 on day 39 and in mouse 8 on day 58 bioluminescence recurred, indicating leukemia relapse which was confirmed by flow cytometry analysis of the BM post mortem. The remaining six mice stayed bioluminescence free until day 130 when the experiment was terminated and no evidence was found for residual KOPN-8 cells in the murine BM by flow cytometry (data not shown).

The time the KOPN-8 bearing mice survived after treatment correlated directly with the course of their bioluminescence signal (Figure 1B). Untreated mice survived on average until day 22 , mice receiving paclitaxel alone until day 30, and mice receiving Moxe alone until day 53. The six mice lacking any bioluminescence after receiving the combination lived until the experiment was terminated on day 130 . We conclude that the combination of paclitaxel and Moxe improves responses in the KOPN8 xenograft model.

\section{$\mathrm{LR}$ is more active than Moxe in vitro}

To test the rIT and paclitaxel combination in an MCL xenograft model, we first compared Moxe and LR for their in vitro activity against MCL cell lines by WST-8 assays. Table 1 shows that LR was more active than Moxe against all MCL cell lines with $\mathrm{IC}_{50} \mathrm{~s}$ ranging from $0.3 \mathrm{ng} /$ $\mathrm{ml}$ to $6 \mathrm{ng} / \mathrm{ml}(6 \mathrm{pmol} / 1$ to $120 \mathrm{pmol} / \mathrm{l})$. The LR-activity was higher by 1.7-fold against Rec-1, 2.4-fold against JeKo-1, 2.4-fold against JVM-2, 3.0-fold against Mino, and 3.3-fold against Z-138 (Table 1). The data show that the deletion of all but the furin cleavage site of domain II results in an increased activity of CD22-targeting rIT against MCL cell lines which is in line with the increased activity of LR against patient derived CLL [8]. 
MCL cell lines need highly variable rIT-exposure time for them to die

Whether the activity of rIT against MCL cells depends on the time the cells are exposed to rIT was tested by treating MCL cell lines with $140 \mathrm{ng} / \mathrm{ml}$ Moxe or equimolar LR for various times, cells were washed and replated in complete medium without rIT and viability determined three days after assay initiation, enough time for the cells to die if they were exposed to a lethal rIT dose. Figure 2 shows that the exposure time needed to kill more than $50 \%$ of the cells varied among the cell lines from less than one hour to three days. Killing of more than $50 \%$ of the Rec- 1 cells was reached after less than 1 hour, of JeKo-1 or JVM-2 cells after 9 hours, of Mino cells after 24 hours and of Z-138 cells after 72 hours. In Rec-1 and

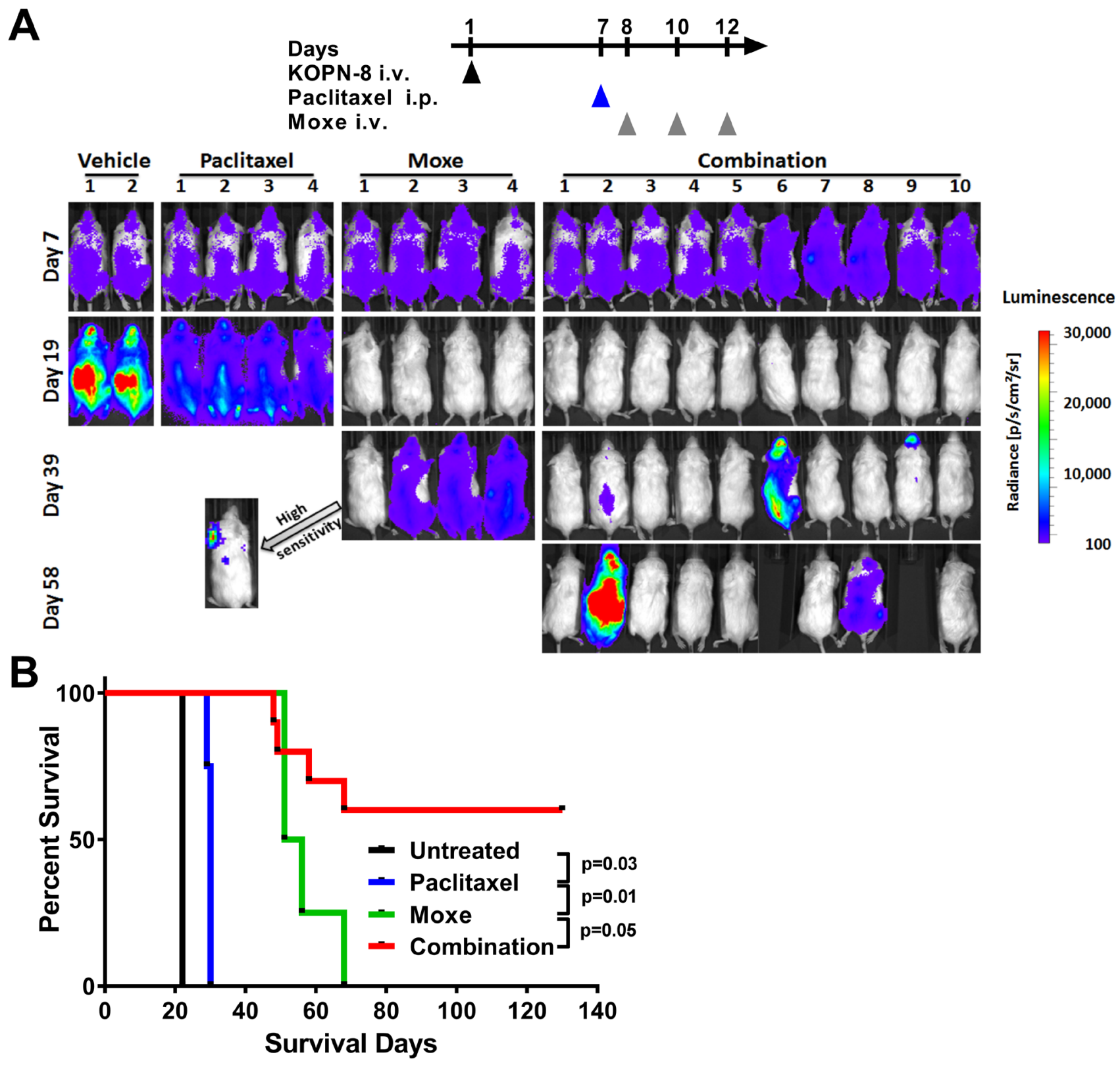

Figure 1:Paclitaxel enhances in vivo efficacy of CD22-targeting immunotoxin in KOPN-8 xenografts. NSG mice bearing luciferase positive KOPN-8 cells were treated with a single dose of $25 \mathrm{mg} / \mathrm{kg}$ paclitaxel i.p. on day 7, 3 doses of $0.4 \mathrm{mg} / \mathrm{kg}$ Moxe QOD from day 8 , or the combination. A. Bioluminescence signal was measured at the indicated days. All images were taken with identical camera settings (binning "medium", exposure time 1 second). Signal intensity is shown as radiance from 100 (blue) to 30,000 (red). Mice without any detectable bioluminescence were imaged a second time using "high sensitivity" camera settings (binning "high", exposure time 1 minute). The arrow points towards the "high sensitivity" image of mouse one of the Moxe group. B. Animal survival is shown as a Kaplan Meyer curve, the significance was determined by log-rank test. 
Table 1: Activity of Moxe and LR against MCL cell lines

\begin{tabular}{|l|c|c|c|c|}
\hline & Moxe-IC50 [ng/ml] & LR-IC50 [ng/ml] & Fold-Difference* & $\boldsymbol{p}$-value \\
\hline Rec-1 & $0.3+/-0.05$ & $0.2+/-0.03$ & 1.7 & 0.08 \\
\hline Jeko-1 & $1.2+/-0.1$ & $0.5+/-0.04$ & 2.4 & 0.002 \\
\hline JVM-2 & $1.6+/-0.2$ & $0.7+/ 0.13$ & 2.4 & 0.01 \\
\hline Mino & $2.6+/-0.8$ & $0.9+/-0.15$ & 3.0 & 0.11 \\
\hline Z-138 & $6.0+/-1.8$ & $1.9+/-0.5$ & 3.3 & 0.10 \\
\hline
\end{tabular}

$\mathrm{IC}_{50}$ was determined by WST8 assay after exposure to serial dilutions of HA22 or LRGGS for 3 days. *Fold-difference of activity was corrected for molecular weight difference between Moxe and LR. Data represents means of three independent experiments, error is shown as SEM, p-values were determined by paired t-tests.

JVM-2 there was no significant difference between Moxe and LR, while JeKo-1 $(p=0.01)$, Mino $(p=0.02)$, and Z-138 $(p=0.03)$ were killed faster by LR than by Moxe. The longer the MCL cells are exposed to rIT in vitro the more likely they die. This exposure time varies from hours to days.

\section{Continuous administration improves responses 10-fold}

Because rITs have a short serum half-life of approximately 15 minutes in mice [8], but JeKo-1 cells need more than 9 hours of rIT exposure for $50 \%$ of the cells to die, we hypothesized that in vivo efficacy may improve by maintaining high rIT blood levels. In mice, continuous administration can be achieved by implanting an osmotic pump into the peritoneal cavity where it remains for seven days at body temperature. We tested LR stability in the standard rIT-formulation PBS at $37^{\circ} \mathrm{C}$. The data in Figure 3A show that LR in PBS loses activity rapidly. LR-activity fell 2.5 -fold below initial activity within one day, fell 9-fold within three days, and was not detectable after seven days. We empirically tested several buffer formulations for their capability to stabilize LR. Stabilization was most efficient in the clinically used Moxe formulation (32 $\mathrm{mM}$ citrate, $0.65 \%$ Tween 80,5 mM EDTA) where LR was stable for up to seven days (Figure 3B). LR-activity on day 1 was similar to the activity at assay initiation and was less than 2-fold reduced by days 3 or 7 , respectively. The buffer exchange from PBS to citrate-buffer itself did not change LR-activity (Supplementary Figure S1). We conclude that LR in the citrate buffer is sufficiently stable to treat mice with a 7-day osmotic pump.

The steady state plasma concentration reached in mice by pump-administrated LR was then determined from plasma serial dilutions using WST-8 assays. Over an 8-day period post-implantation, LR-plasma concentration was on average $45 \mathrm{ng} / \mathrm{ml}$ (Figure 3C) correlating with an area under the curve (AUC) of $350 \mathrm{ng} \mathrm{x}$ day $/ \mathrm{ml}_{\text {Plasma }}$. This steady state plasma concentration was higher than the $\mathrm{IC}_{50}$ of any of the MCL cell lines tested.

A systemic JeKo-1 xenograft model was used to test whether continuously administered LR was more active than bolus doses. Within 14 days after i.v. injection, JeKo1 cells grow predominantly in the BM and proliferate at later stages also in other organs including the spleen and the liver [32]. The data in Figure 3D show that continuous infusion substantially increased efficacy of LR compared to bolus dose administration. At treatment start defined as day 0 , the rate of JeKo-1 BM-infiltration as determined by flow cytometry was on average $38 \%$ and increased to $61 \%$ by day 9 in untreated mice. Three i.v. bolus doses of $2 \mathrm{mg} /$ $\mathrm{kg}$ LR on days 0,2 , and 4 resulted in an infiltration rate of $40 \%$ on day 9 , which was significantly lower than in untreated mice $(p<0.0001)$, but not different from the rate of JeKo-1 BM-infiltration at treatment start. Continuously infused LR reduced the JeKo-1 BM-infiltration by 13-fold to an infiltration rate of $3 \%$ which was significantly lower than the infiltration after three bolus doses $(p<0.0001)$. Maintaining continuously high LR blood levels improved responses greatly.

\section{Enhancement by paclitaxel is minor in vitro but substantial in vivo}

Before testing the combination of pumpadministered LR and paclitaxel in vivo, we determined whether paclitaxel enhances LR in vitro by WST8 assays. Four of five MCL cell lines were sensitive to paclitaxel alone at concentrations achieved in murine organ tissue after a single dose of $25 \mathrm{mg} / \mathrm{kg}$ (Supplementary Figure S2 [33]). The sensitive cell lines showed $\mathrm{IC}_{50} \mathrm{~s}$ of $2.0 \mathrm{nM}$ for JeKo-1, $2.4 \mathrm{nM}$ for Z-138, 4.1 nM for Mino, and 5.8 nM for JVM-2, whereas the less sensitive Rec-1 showed an $\mathrm{IC}_{50}$ of $153 \mathrm{nM}$. When paclitaxel and LR were combined in vitro, activity improved in two of five cell lines (Figure 4A, Supplementary Figure S3). Paclitaxel enhanced LRactivity 3.7-fold against Z-138 and 2.4-fold against Jeko1, while LR-activity against Rec-1, JVM-2, or Mino remained unchanged.

For in vivo testing, JeKo-1-bearing mice were implanted with a 7-day osmotic pump on day 0,14 days after tumor inoculation. To allow for wound healing after the surgery, paclitaxel was injected i.p. 2 days after the pump implantation. Mice were euthanized on day 9 and their BM analyzed. As shown in Figure 4B, mice at treatment start had on average a JeKo-1 BM-infiltration of 
$48 \%$ which increased to $84 \%$ on day 9 in vehicle treated mice. Treatment with paclitaxel alone reduced JeKo-1 BM-infiltration significantly to $76 \%$ on day $9(p=0.004)$. Compared to treatment initiation, the continuously infused LR reduced the JeKo-1 BM-infiltration by 11 -fold ( $p<$ 0.0001). Combining the continuously infused LR with paclitaxel further decreased the Jeko-1 BM-infiltration to $0.1 \%(p<0.0001)$.

To enable a comparison of six independent experiments, we determined the fold-change in JeKo-1 BM infiltration of each of 95 individual mice nine days after treatment initiation relatively to the average JeKo-1 BM infiltration at treatment start within their respective experiment (Figure 4C). The data show that vehicle and Taxol-treated mice progressed, QOD-treated mice showed a stable BM-infiltration, which decreased when LR was administered by pump and decreased even further by the addition of paclitaxel. To directly compare activity of treatment groups, the relative efficacy was mathematically determined (Figures 4D-G). Treating mice with a single dose of paclitaxel on day 2 was on average 1.15-fold more active than vehicle in reducing BM-infiltration on day 9 ( $p$ $=0.05$, Figure 4D). Compared to infiltration at treatment start, three bolus doses LR QOD did not change the JeKo1 BM-infiltration ( $p=0.89$, Figure 4E). Exchanging bolus doses with continuous administration resulted on average in a 14-fold increased efficacy ( $p<0.0001$, Figure 4F). Therefore, a well-tolerated total amount of $84 \mu \mathrm{g}$ LR given by continuous infusion is substantially more active than the $120 \mu \mathrm{g}$ LR given as three bolus doses QOD.

By the addition of paclitaxel to continuously administered LR, the responses improved on average by 135 -fold over pump administration alone $(p=0.0005$, Figure $4 \mathrm{G})$. Of note, in the BM of all mice that had received the combination, viable JeKo-1 cells were still detectable on day 9 by flow cytometry when experiments were terminated. Due to pump properties, the experiments had to be stopped 10 days after implantation and therefore, effects of the treatment on animal survival were not determined. The efficacy of continuously infused LR is substantially enhanced by paclitaxel.

\section{DISCUSSION}

Searching for a molecule that enhances CD22targeting rIT in vivo, we found the combination with

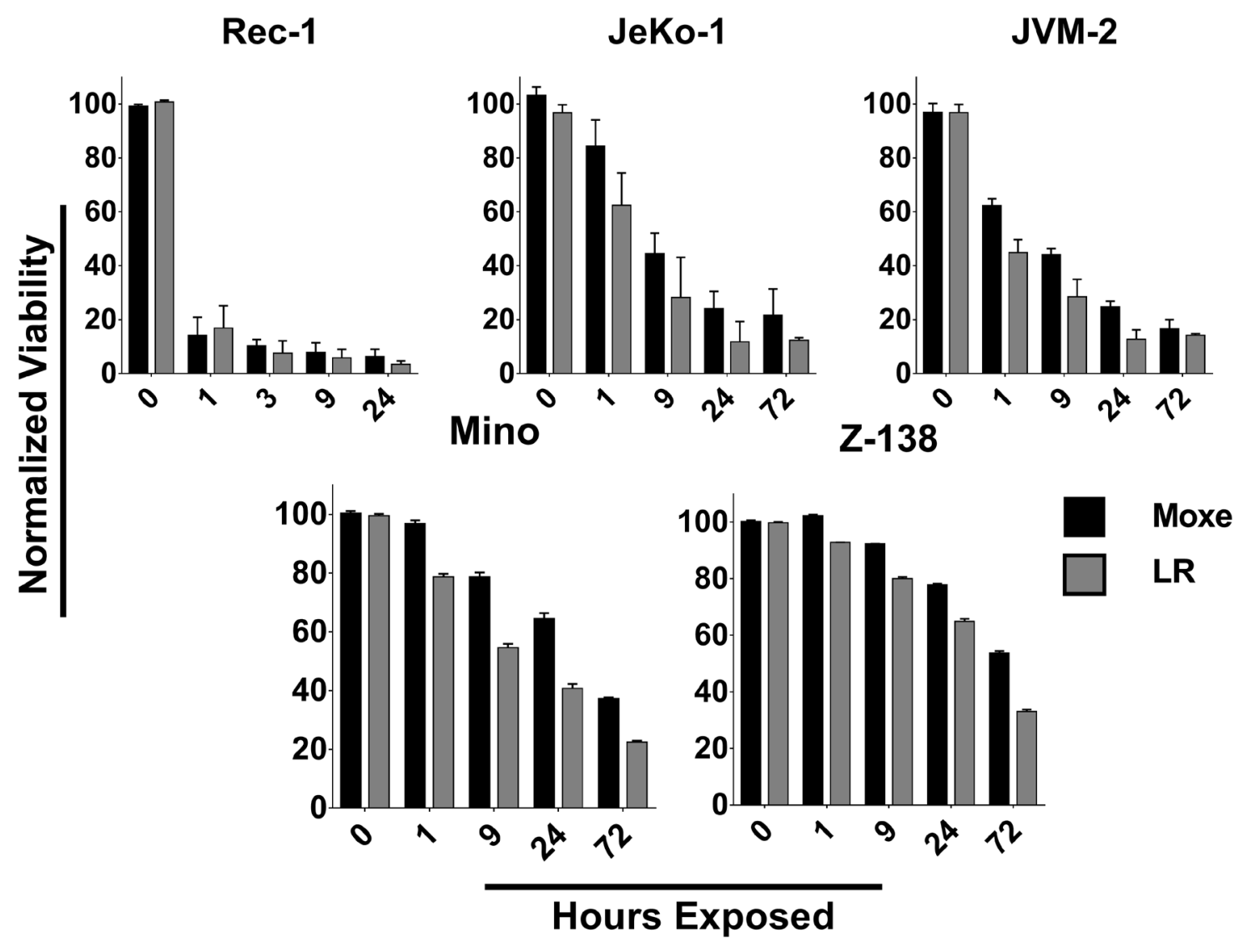

Figure 2: The time for rITs to reach maximal cytotoxic activity is highly variable. Indicated MCL cell lines were exposed to $140 \mathrm{ng} / \mathrm{ml}$ Moxe (black) or equimolar LR (grey) for various times, washed, replated, and cell-viability determined after a total of 3 days. Viability of untreated cells was set to $100 \%, 0 \%$ was defined as real 0 . Each bar represents the average percent living cells of three independent experiments, error is shown as SEM. 

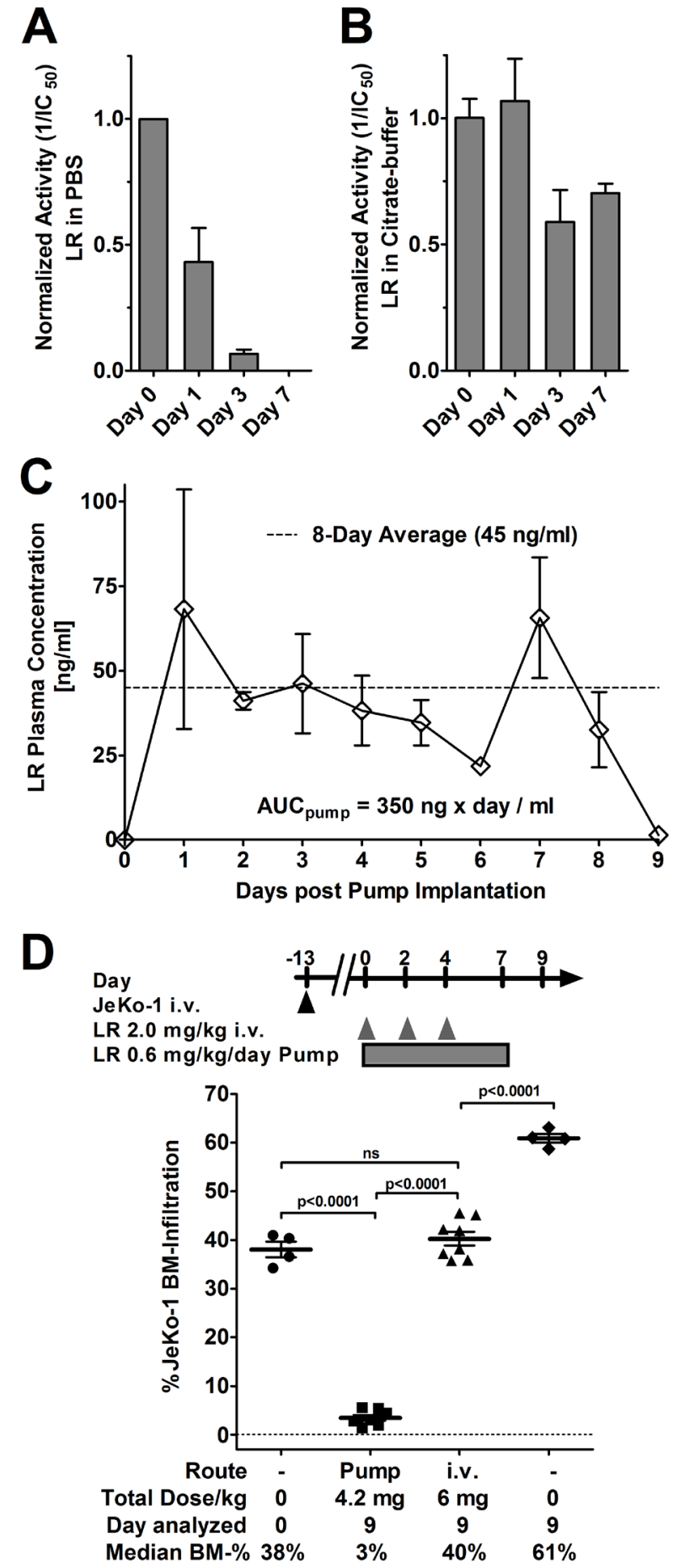

Figure 3: LR in citrate buffer is stable, pump-administration achieves high serum levels increasing activity by 10-fold. LR at $2 \mathrm{mg} / \mathrm{ml}$ in standard buffer PBS A. or citrate buffer B. were incubated at $37^{\circ} \mathrm{C}$ for the indicated times, frozen at $-80^{\circ} \mathrm{C}$, and activity of each sample determined by WST8 assays. Activity of an immediately frozen aliquot (day 0 ) was set to 1 to which the remainder were normalized. Bars indicate average normalized $\mathrm{IC}_{50}$, errors are shown as SEM. C. Mice were implanted with an ALZET osmotic pump containing LR at $1 \mathrm{mg} / \mathrm{ml}$ in citrate buffer on day 0 . From day 1, blood was collected, the plasma separated by centrifugation, and the LR concentration was determined by WST8 assays. Each symbol represents the average LR plasma concentration of 3 mice, except day 6 (only 2 mice), error is shown as SEM (except day 6), AUC as determined by Graph Pad Prism, v.6.01. The average plasma concentration is indicated as a dashed line. D. Some mice bearing systemic Jeko-1 were euthanized on day 0 and the JeKo-1 BM infiltration determined. The remainder were either implanted with a 7-day ALZET osmotic pump containing $1 \mathrm{mg} / \mathrm{ml} \mathrm{LR}$ in citrate buffer and a pump rate of 0.5 $\mu \mathrm{l} / \mathrm{h}$, treated with 3 doses of $2.0 \mathrm{mg} / \mathrm{kg}$ LR QOD i.v. from day 0 , or treated with vehicle. The treated mice were sacrificed on day 9 and their BM was analyzed for JeKo-1 cells by flow cytometry. Symbols indicate individual mice, lines represent mean JeKo-1 BM-infiltration, error as SEM. Significance determined by unpaired t-test as ns (not significant) or as indicated. 
paclitaxel to eradicate leukemia cells in $60 \%$ of KOPN-8bearing mice which survive tumor-free for months. With the goal to test the efficacy of this combination in an MCL xenograft, we found in vitro (i) that LR was more active than Moxe against MCL cells and that (ii) MCL cells had to be exposed to CD22-targeting rITs for a highly variable time for them to die. Due to a short serum half-life of 15 - 20 minutes, rIT plasma concentrations after an i.v. bolus dose fall within less than 2 hours to inactive levels. In accord with the needed exposure time of 9 hours for $50 \%$ of the Jeko- 1 cells to die in vitro, response to CD22targeting rIT improves more than 10-fold in the JeKo-1 xenograft model when i.v. bolus doses are exchanged for continuous infusion by osmotic pumps. The addition of paclitaxel to the optimized continuous rIT-administration increases efficacy more than 100-fold over continuous LR alone.

An in vivo enhancement of mesothelin-targeting rITs by paclitaxel has been described in xenograft models of solid tumors including the mesothelin-expressing pancreatic cancer cell line KLM-1 [28], breast cancer cell line HCC-70, gastric cancer cell line MKN-28 [29], and the cervical cancer cell line KB [31]. Similar to our findings with CD22-targeting rIT, enhancing effects for paclitaxel have been shown for mesothelin-targeting rITs with domain II [31] and without [28, 29], resulting in a new clinical trial testing the efficacy of the LMB100 in combination with Nab-paclitaxel for patients with mesothelin expressing solid tumors (NCT02810418). Taken together the data suggest that paclitaxel enhances rITs in vivo independent from the target or domain IIstatus. In the KOPN-8 model, CD22-targeting rIT alone reduces tumor burden below the detection level by bioluminescence or flow cytometry and only a few cells remain, presumably responsible for late relapse and animal death [24]. It is possible that paclitaxel kills these few remaining cells specifically shown here. An alternative explanation which is supported by the JeKo- 1 data and the data on mesothelin-targeted rITs is that the two drugs have a highly synergistic mechanism in killing the target cells and the few remaining cells in the KOPN-8 model are sensitized to LR.

We currently do not understand the mechanism behind the exceptional in vivo synergy of paclitaxel and rITs. In vivo results with the KB tumors suggested that reduced shedding of surface mesothelin by the paclitaxel treatment reduced decoy receptors produced by the tumor, ultimately increasing delivery of rIT [31]. Improvement of drug delivery after paclitaxel treatment was not shown in a KLM-1 model [28], making the reduction of decoy receptors as the reason for improved rIT-efficacy here unlikely. That only a little CD22 is shed from the cell surface [34] also argues against reduced shedding as a major mechanism for the in vivo enhancement of CD22targeted rIT. While the enhancement by paclitaxel in vivo is substantial, in vitro effects for the enhancement of Mesothelin-targeting rITs have been small or absent
[35]. We similarly find a comparably small two to threefold enhancement of immunotoxin activity against only two of the five MCL cell lines in vitro. Immunotoxins arrest protein synthesis, ultimately leading to a fall of the anti-apoptotic protein MCL1, which destabilizes mitochondria and activates the intrinsic apoptosis [36], while paclitaxel activates the extrinsic apoptosis [37]. Both apoptosis pathways converge in late apoptotic events which may explain the in vitro synergy. Alterations in the expression levels of the regulators of programmed cell death, the Bcl-2 family proteins are common in hematologic malignancies [38] which may explain the variation in the rIT-enhancing effects of paclitaxel in vitro [37]. That the $>100$-fold enhancement of continuously administered LR in JeKo-1-bearing mice by paclitaxel is distinctively higher than the only two-fold enhancement in vitro suggests a distinct mechanism responsible for the exceptional in vivo synergy and indicates that in vitro tests may not be predictive. The in vivo mechanism of the paclitaxel induced rIT-enhancement remains unexplained.

In the first clinical trial, the CD22-targeting rIT BL22 achieved a high rate of objective responses in HCL patients but failed to show activity in CLL, follicular lymphoma, MCL [3], and ALL [4]. Objective responses in ALL patients increased after increasing the affinity to CD22 by 10-fold [7], but were still below the expected response rate [39]. Recent data from ALL xenograft models led to the concept that responses might improve if high rIT blood levels are maintained longer [24]. The previous data suggest that differences in the complex trafficking of Pseudomonas exotoxin A [40] may explain the differences in the time cells need to be exposed to immunotoxin for them to die. We describe here that CD22targeting rITs show an exposure time dependent activity also against MCL cells in vitro and translate these findings into an MCL xenograft model. Because of the rapidly falling rIT blood levels after a bolus dose in patients, the data suggest that responses in B-NHL patients might improve if rIT was given continuously.

In summary, continuous infusion as opposed to bolus doses substantially improves response in an MCL xenograft model which corroborates our previous findings in ALL, emphasizing that a change of the current bolus dose administration to continuous infusion may substantially improve response rates in MCL patients. The exceptionally strong synergy between paclitaxel and CD22-targeting rIT makes this combination a promising candidate for future clinical testing.

\section{MATERIALS AND METHODS}

\section{Reagents}

Moxe [5] and LR [8] were produced as previously described. For the last step in the rIT purification process 
A
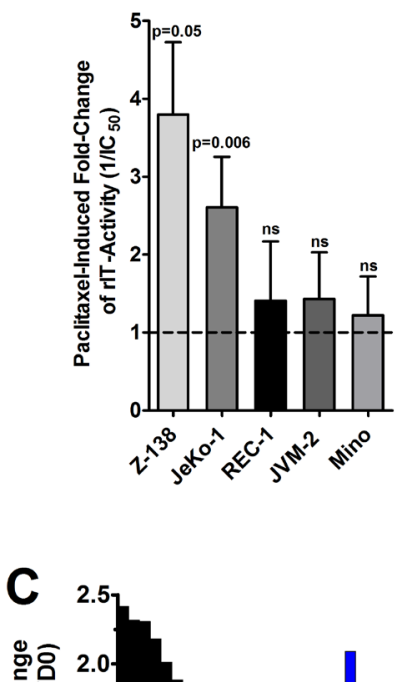

B

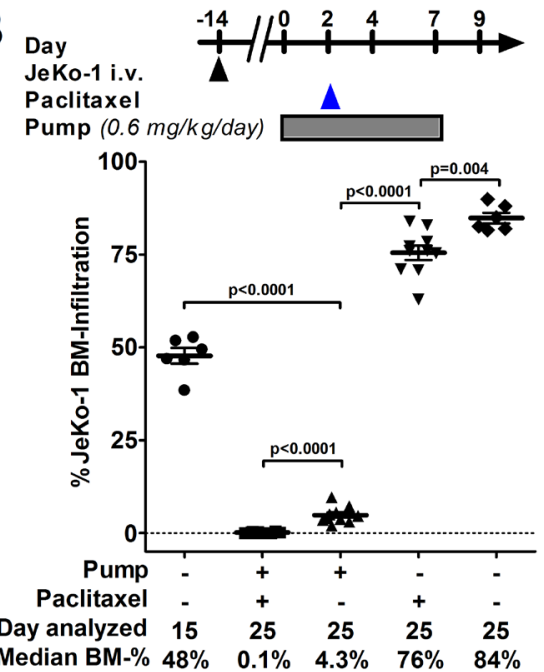

Median BM- $\% \quad 48 \% \quad 0.1 \% \quad 4.3 \% \quad 76 \% \quad 84 \%$
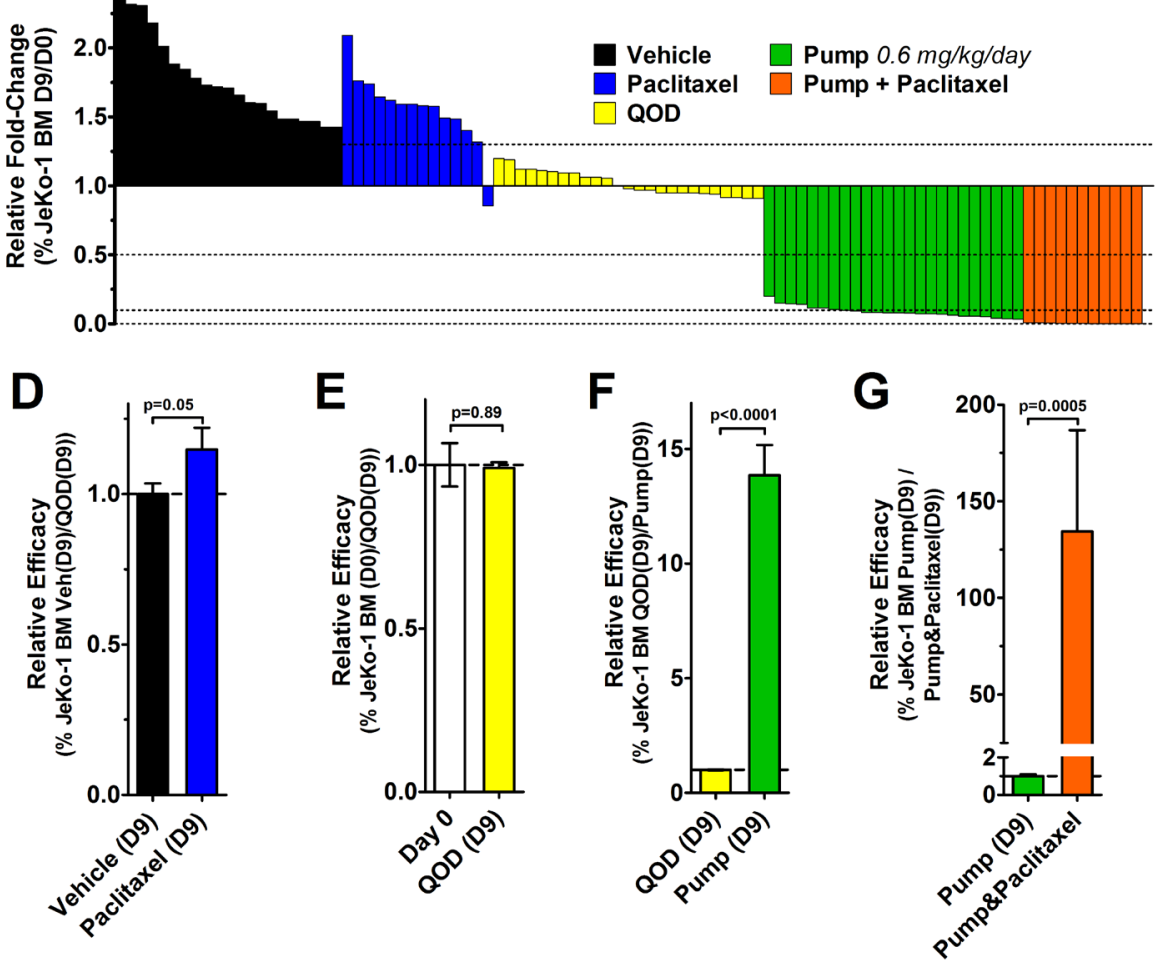

Figure 4: Minor in vitro enhancement contrasts with a substantial improvement of in vivo efficacy by the addition of paclitaxel. A. MCL cell lines were treated with LR and various concentrations of paclitaxel; after 72 hours, cells were stained with Annexin V-PE and 7-AAD and viability was determined by flow cytometry. The bars represent the mean $\mathrm{IC}_{50}$-fold changes when paclitaxel was added. Fold-change values were only included if paclitaxel alone reduced cell viability by at least $15 \%$ (indicating that paclitaxel at this concentration was active) but not more than $85 \%$ (non-linear regression becomes unreliable below this threshold). The fold-changes were summarized from at least three independent experiments; errors as SEM, $\mathrm{p}$-values were determined by unpaired t-tests. B. Some JeKo-1 bearing mice were analyzed for BM infiltration at treatment start on day 0 and the remainder treated with either vehicle, a single dose of $25 \mathrm{mg} / \mathrm{kg}$ paclitaxel i.p. on day 2, implanted with a 7-day ALZET osmotic pump containing $1 \mathrm{mg} / \mathrm{ml} \mathrm{LR}$ in citrate buffer from day 0 , or the combination of pump and paclitaxel. Symbols indicate individual mice, lines represent mean JeKo-1 BM-infiltration, errors as SEM, p-values were determined by unpaired t-test. C. The graph summarizes 95 mice from a total of 6 individual experiments. Each bar represents one mouse and its fold-change of JeKo-1 BM infiltration 9 days after the indicated treatment relative to the average BMinfiltration at the treatment start of the corresponding experiment control on day 0 . The color indicates treatment type as vehicle (black), Taxol mono (blue), $2.0 \mathrm{mg} / \mathrm{kg} \mathrm{LR}$ i.v. QOD (yellow), LR $0.6 \mathrm{mg} / \mathrm{kg} /$ day by 7-day pump (green), and the combination of LR by pump and paclitaxel (red). D.-G. To determine the relative efficacy, the average BM-infiltration in the indicated groups was set to 1 and the relative fold-change was determined as (D) vehicle treated on day 9 (D9) vs. paclitaxel (D9), as (E) day 0 vs. QOD (D9), (F) as QOD (D9) vs. pump-administrated LR (D9), and as (G) pump-administrated LR without vs. with paclitaxel (D9). Color codes in (D-G) are as indicated in (C), the bars show the mean relative fold-change, errors are shown as SEM, p-values were determined by unpaired t-tests. 
[42], the size exclusion chromatography, elution buffer PBS, pH 7.4 was changed to a citrate formulation $(32 \mathrm{mM}$ citrate pH 6.0, 5 mM EDTA, 0.65\% Tween 80). Clinical grade paclitaxel in Cremophore $(6 \mathrm{mg} / \mathrm{ml})$ was purchased from Teva Pharmaceuticals (\#00703-4764-01). For in vitro application, paclitaxel was diluted in RPMI to indicated concentrations and for in vivo application it was diluted to $1.25 \mathrm{mg} / \mathrm{ml}$ in PBS, $\mathrm{pH} 7.4$.

\section{Cell lines}

The Jeko-1, JVM-2, and REC-1 cells were described previously [43]. The Mino and Z-138 cells were kindly provided by Dr. Louis Staudt, NCI/NIH. The B-cell precursor (BCP-) ALL cell line KOPN-8 [24] was transduced with a luciferase and GFP containing lentivirus, sorted for the top 5\% GFP expression twice, and singlecloned. All cells were grown in RPMI supplemented with $10 \%$ fetal bovine serum, $100 \mathrm{U}$ penicillin, and $100 \mathrm{mg}$ streptomycin (Invitrogen).

\section{Cell assays}

Cytotoxicity was determined by WST-8 (Dojindo Molecular Technologies, Rockville, MD, \#CK04-20) as described [26]. 5,000 cells/well were incubated with various rIT concentrations for 72 hours. WST- 8 reagent was added, absorbance was measured 2 hours later, values were normalized between Cycloheximide $(10 \mu \mathrm{g} / \mathrm{ml}$ final, Sigma-Aldrich, St. Louis, MO, \#C4859-1ML) and untreated control and, non-linear regression to obtain $\mathrm{IC}_{50}$ concentrations was done using GraphPad Prism v6.01.

For in vitro apoptosis assays by flow cytometry, 1 million cells $/ \mathrm{ml}$ were incubated with $2.8 \mathrm{nM}$ rIT for various times, cells washed twice after the indicated incubation times, resuspended in complete RPMI, and transferred to a new plate. Seventy-two hours after assay initiation, cells were stained with 7-AAD/Annexin-PE, and measured with a FACS Calibur (BD). Results were analyzed with FlowJo software (Tree Star).

\section{Stability testing}

LR in PBS or in Citrate buffer was concentrated to $1 \mathrm{mg} / \mathrm{ml}$ using Amicon Ultracell spin columns (Millipore, \#UFC803024). To test for stability at $37^{\circ} \mathrm{C}$, tubes were incubated in a heating block for indicated times and aliquots frozen at $-80^{\circ} \mathrm{C}$ until used. The remaining rITactivity was determined by WST8 assays.

\section{Animal studies}

Animals were handled according to NIH guidelines; studies were approved by the NCI Animal Care and Use Committee.
Ten million JeKo-1 or five million KOPN-8 cells were injected on Day 1 via tail vein into 6- to 8-week-

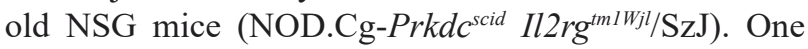
$\mathrm{mg} / \mathrm{ml} \mathrm{LR}$ in citrate buffer was loaded into 7-day ALZET osmotic pumps dispensing at $0.5 \mu \mathrm{l} / \mathrm{h}$. The pumps were surgically implanted into the peritoneal cavity following manufacturer's instructions. rIT was i.v. injected as three bolus doses of $0.4 \mathrm{mg} / \mathrm{kg}$ Moxe QOD or $2.0 \mathrm{mg} / \mathrm{kg} \mathrm{LR}$ QOD. Paclitaxel was given as a single dose of $25 \mathrm{mg} / \mathrm{kg}$ paclitaxel i.p. To assess treatment responses, mice were euthanized 10 days after osmotic pump implantation. BM was extracted by flushing femurs. Mouse-derived tissue was $\mathrm{F}_{\mathrm{c}}$-receptor blocked with anti-murine CD16/32, JeKo-1 cells were stained with anti-human-CD20-FITC, viability was determined using 7-AAD, and cells were analyzed on a FACS Calibur.

To measure the rIT plasma concentration, mice were implanted with osmotic pumps and two groups of three mice each were bled alternatingly QOD by puncture of the mandibular vein. Thirty $\mu 1$ blood/mouse/puncture was collected in heparinized tubes, plasma separated by centrifugation, and stored at $-80{ }^{\circ} \mathrm{C}$ until used. Thawed, plasma samples were serial diluted in RPMI and tested for cytotoxic activity against Rec-1 cells by WST-8. The plasma dilution factor at which $50 \%$ Rec-1 cells were growth inhibited was determined by non-linear regression and the corresponding LR plasma concentration was extrapolated from a standard curve generated by treating Rec-1 with serial dilutions of LR.

\section{Luciferase imaging}

D-luciferin (150 mg/kg VivoGlo, Promega, \# P1043) was injected i.p., mice anesthetized with Isoflurane, and images taken 5 minutes after injection using a Xenogen IVIS-100 (Caliper).

\section{Statistics}

Statistical analyses were performed with Graph Pad Prism v6.01 as paired or unpaired t-tests as indicated and as log-rank tests for animal survival.

\section{Abbreviations}

ALL- Acute lymphoblastic leukemia, AUC-area under the curve, B-ALL-B-cell ALL, BCP-ALL-B-cell precursor ALL, BM- bone marrow , B-NHL-B cell non Hodgkin's lymphoma, BTK-Bruton's Tyrosine kinase , CLL-chronic lymphocytic leukemia, GFP-green fluorescent protein, HCL- hairy cell leukemia, MCLmantle cell lymphoma, Moxe-Moxetumomab pasudotox, QOD-every other day, rIT-recombinant immunotoxin. 


\section{Author contributions}

F.M., T.C., and S.S. performed the experiments, F.M. and I.P. supervised, analyzed and interpreted the experiments and wrote the manuscript.

\section{ACKNOWLEDGMENTS}

The content is solely the responsibility of the authors and does not necessarily represent the official views of the National Cancer Institute or the National Institutes of Health. The authors wish to thank Dr. Louis Staudt for providing MCL cell lines Z-138 and Mino, the team of the building 37 animal facility, and Dr. Bih-Rong Wei for technical support with the bioluminescence measurements. F.M. was supported in part by the German Research Foundation, award number MU 3619/1-1.

\section{CONFLICTS OF INTEREST}

I.P. is a co-inventor on patents assigned to the NIH for the investigational products. F.M., T.C., S.S. and I.P. declare no financial interests.

\section{FUNDING}

The work was supported in part by the Intramural Research Program of the NIH, National Cancer Institute, Center for Cancer Research, and with a Cooperative Research and Development Agreement (\#1975) with Medimmune, LLC. F.M. was supported in part by the German Research Foundation, award number MU 3619/11.

\section{REFERENCES}

1. Pastan I, Hassan R, Fitzgerald DJ, Kreitman RJ. Immunotoxin therapy of cancer. Nat Rev Cancer. 2006; 6:559-565.

2. Mansfield E, Amlot P, Pastan I, FitzGerald DJ. Recombinant RFB4 immunotoxins exhibit potent cytotoxic activity for CD22-bearing cells and tumors. Blood. 1997; 90:2020-2026.

3. Kreitman RJ, Squires DR, Stetler-Stevenson M, Noel P, FitzGerald DJ, Wilson WH, Pastan I. Phase I trial of recombinant immunotoxin RFB4(dsFv)-PE38 (BL22) in patients with B-cell malignancies. J Clin Oncol. 2005; 23:6719-6729.

4. Wayne AS, Kreitman RJ, Findley HW, Lew G, Delbrook C, Steinberg SM, Stetler-Stevenson M, Fitzgerald DJ, Pastan I. Anti-CD22 immunotoxin RFB4(dsFv)-PE38 (BL22) for CD22-positive hematologic malignancies of childhood: preclinical studies and phase I clinical trial. Clin Cancer Res. 2010; 16:1894-1903.
5. Salvatore G, Beers R, Margulies I, Kreitman RJ, Pastan I. Improved cytotoxic activity toward cell lines and fresh leukemia cells of a mutant anti-CD22 immunotoxin obtained by antibody phage display. Clin Cancer Res. 2002; 8:995-1002.

6. Kreitman RJ, Tallman MS, Robak T, Coutre S, Wilson WH, Stetler-Stevenson M, Fitzgerald DJ, Lechleider R, Pastan I. Phase I trial of anti-CD22 recombinant immunotoxin moxetumomab pasudotox (CAT-8015 or HA22) in patients with hairy cell leukemia. J Clin Oncol. 2012; 30:1822-1828.

7. Wayne AS, Shah NN, Bhojwani D, Silverman LB, Whitlock JA, Stetler-Stevenson M, Kreitman RJ, Goswami T, Ibrahim R, Pastan I. Pediatric phase 1 trial of moxetumomab pasudotox: Activity in chemotherapy refractory acute lymphoblastic leukemia (ALL). Cancer Res. 2014; 74(19 Supplement):CT230-CT230.

8. Weldon JE, Xiang L, Chertov O, Margulies I, Kreitman RJ, FitzGerald DJ, Pastan I. A protease-resistant immunotoxin against CD22 with greatly increased activity against CLL and diminished animal toxicity. Blood. 2009; 113:37923800 .

9. Liu W, Onda M, Lee B, Kreitman RJ, Hassan R, Xiang L, Pastan I. Recombinant immunotoxin engineered for low immunogenicity and antigenicity by identifying and silencing human B-cell epitopes. Proc Natl Acad Sci U S A. 2012; 109:11782-11787.

10. Mazor R, Vassall AN, Eberle JA, Beers R, Weldon JE, Venzon DJ, Tsang KY, Benhar I, Pastan I. Identification and elimination of an immunodominant T-cell epitope in recombinant immunotoxins based on Pseudomonas exotoxin A. Proc Natl Acad Sci U S A. 2012; 109:E35973603.

11. Avivi I, Goy A. Refining the mantle cell lymphoma paradigm: impact of novel therapies on current practice. Clin Cancer Res. 2015; 21:3853-3861.

12. Wang $\mathrm{ML}$, Lee $\mathrm{H}$, Chuang $\mathrm{H}$, Wagner-Bartak $\mathrm{N}$, Hagemeister F, Westin J, Fayad L, Samaniego F, Turturro F, Oki Y, Chen W, Badillo M, Nomie K, et al. Ibrutinib in combination with rituximab in relapsed or refractory mantle cell lymphoma: a single-centre, open-label, phase 2 trial. Lancet Oncol. 2016; 17:48-56.

13. Dreyling M, Jurczak W, Jerkeman M, Silva RS, Rusconi C, Trneny M, Offner F, Caballero D, Joao C, Witzens-Harig M, Hess G, Bence-Bruckler I, Cho SG, et al. Ibrutinib versus temsirolimus in patients with relapsed or refractory mantle-cell lymphoma: an international, randomised, openlabel, phase 3 study. Lancet. 2016; 387:770-778.

14. Baiocchi RA, Alinari L, Lustberg ME, Lin TS, Porcu P, Li X, Johnston JS, Byrd JC, Blum KA. Phase 2 trial of rituximab and bortezomib in patients with relapsed or refractory mantle cell and follicular lymphoma. Cancer. 2011; 117:2442-2451.

15. Coiffier B, Osmanov EA, Hong X, Scheliga A, Mayer J, Offner F, Rule S, Teixeira A, Walewski J, de Vos S, 
Crump M, Shpilberg O, Esseltine DL, et al. Bortezomib plus rituximab versus rituximab alone in patients with relapsed, rituximab-naive or rituximab-sensitive, follicular lymphoma: a randomised phase 3 trial. Lancet Oncol. 2011; 12:773-784.

16. Robak T, Huang H, Jin J, Zhu J, Liu T, Samoilova O, Pylypenko H, Verhoef G, Siritanaratkul N, Osmanov E, Alexeeva J, Pereira J, Drach J, et al. Bortezomib-based therapy for newly diagnosed mantle-cell lymphoma. N Engl J Med. 2015; 372:944-953.

17. Goy A, Sinha R, Williams ME, Kalayoglu Besisik S, Drach J, Ramchandren R, Zhang L, Cicero S, Fu T, Witzig TE. Single-agent lenalidomide in patients with mantlecell lymphoma who relapsed or progressed after or were refractory to bortezomib: phase II MCL-001 (EMERGE) study. J Clin Oncol. 2013; 31:3688-3695.

18. Wang M, Fayad L, Wagner-Bartak N, Zhang L, Hagemeister F, Neelapu SS, Samaniego F, McLaughlin P, Fanale M, Younes A, Cabanillas F, Fowler N, Newberry $\mathrm{KJ}$, et al. Lenalidomide in combination with rituximab for patients with relapsed or refractory mantle-cell lymphoma: a phase 1/2 clinical trial. Lancet Oncol. 2012; 13:716-723.

19. Ruan J, Martin P, Shah B, Schuster SJ, Smith SM, Furman RR, Christos P, Rodriguez A, Svoboda J, Lewis J, Katz O, Coleman M, Leonard JP. Lenalidomide plus Rituximab as initial treatment for mantle-cell lymphoma. N Engl J Med. 2015; 373:1835-1844.

20. Ansell SM, Tang H, Kurtin PJ, Koenig PA, Inwards DJ, Shah K, Ziesmer SC, Feldman AL, Rao R, Gupta M, Erlichman C, Witzig TE. Temsirolimus and rituximab in patients with relapsed or refractory mantle cell lymphoma: a phase 2 study. Lancet Oncol. 2011; 12:361-368.

21. Cheah CY, Seymour JF, Wang ML. Mantle cell lymphoma. J Clin Oncol. 2016; 34(11):1256-1269.

22. Campo E, Rule S. Mantle cell lymphoma: evolving management strategies. Blood. 2015; 125:48-55.

23. Dreyling M, Ferrero S, Hermine O. How to manage mantle cell lymphoma. Leukemia. 2014; 28:2117-2130.

24. Mueller F, Cunningham T, Liu X, Wayne AS, Pastan I. Wide variability in the time required for immunotoxins to kill B lineage acute lymphoblastic leukemia cells: Implications for trial design. Clin Cancer Res. 2016; 22:4913-4922.

25. Ali-Rahmani F, FitzGerald DJ, Martin S, Patel P, Prunotto M, Ormanoglu P, Thomas C, Pastan I. Anticancer effects of mesothelin-targeted immunotoxin therapy are regulated by tyrosine kinase DDR1. Cancer Res. 2016; 76:1560-1568.

26. Liu X, Mueller F, Wayne AS, Pastan I. Protein kinase inhibitor H89 enhances the activity of Pseudomonas exotoxin A-based immunotoxins. Mol Cancer Ther. 2016; 15:1053-1062.

27. Antignani A, Sarnovsky R, FitzGerald DJ. ABT-737 promotes the dislocation of ER luminal proteins to the cytosol, including pseudomonas exotoxin. Mol Cancer
Ther. 2014; 13:1655-1663.

28. Hollevoet K, Mason-Osann E, Liu XF, Imhof-Jung S, Niederfellner G, Pastan I. In vitro and in vivo activity of the low-immunogenic antimesothelin immunotoxin RG7787 in pancreatic cancer. Mol Cancer Ther. 2014; 13:2040-2049.

29. Alewine C, Xiang LM, Yamori T, Niederfellner G, Bosslet K, Pastan I. Efficacy of RG7787, a next-generation mesothelin-targeted immunotoxin, against triple-negative breast and gastric cancers. Mol Cancer Ther. 2014; 13:2653-2661.

30. Kolyvas E, Rudloff M, Poruchynsky M, Landsman R, Hollevoet K, Venzon D, Alewine C. Mesothelin-targeted immunotoxin RG7787 has synergistic anti-tumor activity when combined with taxanes. Oncotarget. 2017; 8:91899199. doi: 10.18632/oncotarget.13984.

31. Zhang YJ, Xiang LM, Hassan R and Pastan I. Immunotoxin, Taxol synergy results from a decrease in shed mesothelin levels in the extracellular space of tumors. Proc Natl Acad Sci U S A. 2007; 104:17099-17104.

32. Klanova M, Soukup T, Jaksa R, Molinsky J, Lateckova L, Maswabi BC, Prukova D, Brezinova J, Michalova K, Vockova P, Hernandez-Ilizaliturri F, Kulvait V, Zivny J, et al. Mouse models of mantle cell lymphoma, complex changes in gene expression and phenotype of engrafted MCL cells: implications for preclinical research. Lab Invest. 2014; 94:806-817.

33. Innocenti F, Danesi R, Di Paolo A, Agen C, Nardini D, Bocci G, Del Tacca M. Plasma and tissue disposition of paclitaxel (taxol) after intraperitoneal administration in mice. Drug metabolism and disposition. 1995; 23:713-717.

34. Shah NN, Stevenson MS, Yuan CM, Richards K, Delbrook C, Kreitman RJ, Pastan I, Wayne AS. Characterization of CD22 expression in acute lymphoblastic leukemia. Pediatr Blood Cancer. 2015; 62:964-969.

35. Alewine CC, Kolyvas E, Boslett K, Pastan I. Combination of taxanes with mesothelin-targeted immunotoxin RG7787 induces synergistic killing of pancreatic cancer. AACR; Cancer Res. 2015; 75:Abstract nr 2566.

36. Du X, Youle RJ, FitzGerald DJ, Pastan I. Pseudomonas exotoxin A-mediated apoptosis is Bak dependent and preceded by the degradation of Mcl-1. Mol Cell Biol. 2010; 30:3444-3452.

37. Wang S, Wang Z, Dent P, Grant S. Induction of tumor necrosis factor by bryostatin 1 is involved in synergistic interactions with paclitaxel in human myeloid leukemia cells. Blood. 2003; 101:3648-3657.

38. Reed JC. Bcl-2-family proteins and hematologic malignancies: history and future prospects. Blood. 2008; 111:3322-3330.

39. Mussai F, Campana D, Bhojwani D, Stetler-Stevenson M, Steinberg SM, Wayne AS, Pastan I. Cytotoxicity of the anti-CD22 immunotoxin HA22 (CAT-8015) against paediatric acute lymphoblastic leukaemia. Br J Haematol. 
2010; 150:352-358.

40. Moreau D, Kumar P, Wang SC, Chaumet A, Chew SY, Chevalley H, Bard F. Genome-wide RNAi screens identify genes required for Ricin and PE intoxications. Dev Cell. 2011;21:231-244.

41. Pasetto M, Antignani A, Ormanoglu P, Buehler E, Guha R, Pastan I, Martin SE, FitzGerald DJ. Whole-genome RNAi screen highlights components of the endoplasmic reticulum/ Golgi as a source of resistance to immunotoxin-mediated cytotoxicity. Proc Natl Acad Sci U S A. 2015; 112:E11351142.

42. Pastan I, Beers R, Bera TK. Recombinant immunotoxins in the treatment of cancer. Methods Mol Biol. 2004; 248:503518.

43. Baskar S, Wiestner A, Wilson WH, Pastan I, Rader C. Targeting malignant $\mathrm{B}$ cells with an immunotoxin against ROR1. MAbs. 2012; 4:349-361. 